\title{
PRIORITIES FOR COMMUNICABLE DISEASE CONTROL IN NEW SOUTH WALES, 2003
}

\section{Jeremy McAnulty and Kim Stewart, on behalf of the NSW Public Health Network \\ Centre for Health Protection \\ NSW Department of Health}

This article describes the priorities for the control of communicable disease in New South Wales from 2003.

\section{SUMMARY}

The control of communicable diseases is a core function of government, which must be achieved through collaboration with clinicians, laboratory scientists, affected communities, and other government and community-based agencies. Changes in environmental and social conditions, and the development of new prevention technologies, influence the incidence of communicable diseases and the community's ability to control them. The capacity of NSW Health to control communicable diseases also depends on maintaining a strong network of skilled public health professionals.

From 2003, the proposed 'top 10' priorities for communicable disease surveillance, prevention, and control in New South Wales are:

- disease outbreaks, including those caused by emerging diseases and the deliberate release of biological agents;

- blood-borne pathogens (focussing on HIV, hepatitis C and hepatitis B);

- sexually transmissible infections (focussing on HIV, chlamydia, syphilis, and gonorrhoea);

- vaccine preventable diseases (focussing on measles, congenital rubella, invasive pneumococcal disease, invasive Haemophilus influenza type b disease, pertussis, and influenza);

- tuberculosis;

- enteric disease;

- meningococcal disease;

- mosquito-borne diseases caused by flaviviruses;

- infections caused by antibiotic resistant organisms;

- infections associated with health care.

\section{BACKGROUND}

Throughout history, changes in the environment have presented new threats to human health. When humans lived in small tribal groups, the effects of any new communicable diseases were probably limited by the small size and relative isolation of the group. After causing illness in members of the groups, a newly-introduced communicable disease would probably have burnt itself out as it ran out of susceptible victims. As humans moved into larger community groups, the number of susceptible people in each group increased, often with little in the way of water, sewerage, and social infrastructure to support them. Crowding and squalid living conditions presented opportunities for airborne pathogens to spread readily, and when communal water became contaminated with sewerage, massive outbreaks of enteric disease occurred. As contact between communities grew, through trade or colonisation or conflict, previously isolated communities were exposed to new organisms carried by traders, colonisers, and soldiers, or by the vermin that accompanied them.

\section{Community solutions}

From ancient times, communities have developed public services designed to improve living conditions that, in turn, have helped prevent disease. These services have included the provision of potable drinking water and sewerage systems, the drainage of swamps, and quarantine. A range of diverse social policies including public education, welfare, and town planning, contributed to reducing the transmission opportunities for agents of communicable disease.

\section{Public health}

Only in the last 200 years or so has the understanding of the germ theory and the importance of some of the most important yet basic public health measures, such as availability of running water and hand-washing, been recognised. The development of immunisation, first against smallpox, and later for a growing number of other diseases including diphtheria, pertussis, tetanus, polio, and measles, led to massive declines in morbidity and mortality associated with these conditions.

\section{Epidemiology}

Epidemiological methods for the surveillance, investigation, and control of diseases began with the collection of basic demographic statistics including deaths and their causes. Since then, epidemiology has advanced to harness a wide range of data sources for disease (including mandatory disease notifications and statistical collections on hospitalisations and death), risk (including behavioural, social, and environmental factors), sophisticated analytical study designs (for example, cohort and case-control studies), and meta-analyses that sometimes expose subtle disease risks. An improved understanding of risks enabled the development of better strategies for disease prevention.

\section{Treatments}

Antibiotics emerged relatively recently in the treatment of disease. A person's immune system has always been the 
mainstay against infection, assisted by the occasional surgical intervention to drain pus or remove gangrenous body parts. Antibiotic treatment only became available on a limited basis during World War II, and has had a dramatic effect on the outcome of a wide range of bacterial and parasitic infections. However, specific treatments remain elusive for most viral infections, and resistance to antibiotics has emerged for many bacterial infections.

\section{Social challenges}

Despite technological advances in epidemiology, prevention, control, and treatments, disparities in health status in general-and the incidence of communicable diseases in particular - are still related to poverty and access to services. Certain groups are at increased risk of communicable disease, either because of susceptibility (for example, immunocompromising conditions), age, sex, occupation, behaviours, living conditions, nutrition, or access to services. For example: meat workers are at increased risk of Q fever; people who have lived in countries with high rates of tuberculosis are at increased risk of that disease; people who live in rural NSW are at increased risk of arbovirus infections; men who have sex with men have an increased risk of HIV infection; injecting drug users are at increased risk of blood-borne infections such as hepatitis $\mathrm{C}$; and children who have not been immunised are at increased risk of measles. The disadvantaged, refugees, and indigenous communities, have higher rates of a range of communicable diseases than does the general community. The recognition that certain factors are associated with certain risks can allow for the development of targeted prevention and control measures.

\section{Environmental challenges}

Growing populations create new environmental demands. For example, if new land opened up for housing developments contains wetland areas, this can expose new residents to mosquito-borne diseases. Inadequate disposal of sewerage may lead to the contamination of estuarine waters, and the shellfish living in them, with agents of communicable disease such as Norovirus and hepatitis A.

\section{New technologies}

New technologies can bring immense benefits, but sometimes carry health risks. Examples include the invention of cooling-tower-based air conditioning systems, which inadvertently provide a breeding ground for Legionella bacteria and subsequent outbreaks of legionnaires disease. The development of mass food production has occasionally led to mass food poisoning. Changes in the way animals are farmed and fed have been linked to the outbreak of bovine spongiform enceph- alopathy ('mad cow' disease) and the subsequent outbreak of the human variant, Creutzfeldt-Jakob disease, in Europe. International air travel now presents the potential for the carriage of exotic diseases, such as Severe Acute Respiratory Syndrome, rapidly across the globe. Improved laboratory technologies have allowed the development of agents of biological warfare, such as the powdered anthrax that was disseminated through mail in the United States in 2001.

New technologies in health care have also had their downsides. Mass treatments with reusable, non-sterile syringes and needles probably contributed to the spread of blood-borne diseases like hepatitis $\mathrm{C}$, hepatitis $\mathrm{B}$, and HIV in some countries, as did blood transfusion before the introduction of screening. The use of immunosuppressive drugs to treat various medical conditions, and the increase in the older population, have meant that more people are vulnerable to infections. The congregation of susceptible and sick people in hospitals and nursing homes facilitates the transmission of infection. The widespread use of antibiotics to treat infection has led to the natural selection of antibioticresistant bacteria within populations, making treatment more difficult.

\section{Complacency}

The very success of public health interventions is in some ways a threat to their continuation. Because immunisation has practically eliminated many diseases, few Australians are aware of the horrors of diseases like polio and diphtheria; consequently, there is a risk of loss of public support for immunisation programs. Similarly, because new treatments for HIV disease have made the development of AIDS less common in people infected with HIV, there is a risk that preventive safe-sex messages will lose their impact. Because programs providing sterile needles and syringes have been so successful, there are few HIV infections among injecting drug users and there is risk that these programs will be seen as expendable. The decline of tuberculosis treatment services in New York was directly implicated in a resurgence of the disease in that city during the 1980 s.

The challenge for public health is to identify, foresee, and respond to new threats as they emerge, while maintaining existing control measures. It is therefore important to regularly review disease control programs to ensure that they focus on minimising the risk posed by threats to public health.

\section{A SKILLED WORKFORCE}

The control of communicable diseases depends on the coordination and effective functioning of a network of 
public health practitioners. Issues that influence this effective functioning include:

- maintaining high skills among members of the network;

- supporting the work of the network through guidelines, ready access to expert advice, data systems, and administrative and quality improvement programs;

- effective communication within the network, and to key stakeholders, including health-care providers and the broader community;

- capacity to respond rapidly to emergencies, and using network capacity to address crises as they emerge.

\section{NSW STRUCTURES}

In New South Wales, communicable disease prevention is coordinated primarily through the public health network of local public health units, and the NSW Department of Health's Centre for Health Protection, in partnership with diagnostic laboratories and clinicians. At a local level, public health units work closely with other employees of the area health services, providers of primary and specialist health care, other governmental and non-government agencies, and the community, to ensure that control programs are successfully implemented. At the state level, the Centre for Health Protection (particularly the Communicable Diseases and AIDS-Infectious Diseases and Environmental Health Branches, and the soon to be devolved Food Branch) monitors health indicators and develops policies and programs, based on available evidence, in consultation with a range of advisory committees and other agencies.

\section{THE IMPORTANCE OF DATA}

The control of communicable diseases requires both data inputs and information outputs. Data inputs include:

- disease surveillance (monitoring diseases and the detection of outbreaks);

- data on policy implementation (monitoring whether control and prevention programs are put in place);

- investigations (analysing surveillance data, conducting research and investigating outbreaks to identify disease risk factors);

- the scientific literature (monitoring newly identified risks and control measures from around the world);

- intelligence garnered from reports by public health units, and other states and territories (through the Communicable Diseases Network of Australia);

- intelligence derived from international e-mail bulletin boards (such as ProMed);

- data from other agencies on disease threats (reporting diseases in animals, or environmental contamination);
- expert opinion derived from advisory committees. Key Departmental committees include the Public Health Nurses and Surveillance Officers Group, the Public Health Directors Forum, the Meningococcal Diseases Advisory Committee, the Tuberculosis Advisory Committee, the Ministerial Advisory Committee on AIDS Strategy, the Ministerial Advisory Committee on Hepatitis, the Infection Control Advisory Group, the Immunisation Advisory Committee, and the Sexual Health Advisory Committee.

Information outputs include:

- production of the Notifiable Diseases Manual (which includes protocols for public health units on how to control transmission following the notification of a case);

- regular publication of surveillance data in the NSW Public Health Bulletin, in local newsletters produced by public health units, on the internet, and in the medical literature;

- dissemination of control policies, guidelines, recommendations and fact sheets (through NSW Health Circulars, the NSW Public Health Bulletin, letters to area health services, general practitioners, health care workers and schools, the websites of the NSW Department of Health and the area health services, and telephone hot lines);

- contribution to state and national policies on disease control produced by other branches of the NSW Department of Health and other agencies;

- provision of advice to clinicians;

- health alerts and advice issued through the media;

- training of public health professionals in the methods of disease control.

\section{DEVELOPING PRIORITIES}

In 2000, NSW Health developed a list of draft priorities for disease control. These priorities included the elimination of measles, congenital rubella, and congenital syphilis; monitoring risk factors for new hepatitis $\mathrm{C}$ infections; gaining a better understanding of risk factors for invasive pneumococcal disease and infections associated with health care; and minimising the incidence and management of multi-drug resistant tuberculosis.

There have been several key developments relevant to communicable disease control since 2000 that affect priorities in NSW. Positive developments include:

- increasing rates of immunisation against childhood diseases;

- a decline in reports of new AIDS cases and deaths, primarily due to better treatments for HIV;

- the development of a new state agency for food safety (SafeFood NSW) and Commonwealth funding for 
enhanced surveillance of foodborne disease through OzFoodNet;

- funding from the Commonwealth Department of Health and Ageing for targeted immunisation against pneumococcal disease in infants at high risk;

- the introduction of a childhood and school-based vaccination program against meningococcal serogroup C disease;

- the introduction of a mandatory statewide monitoring system for infections associated with healthcare.

On the other hand, threats that have emerged or continued include:

- severe acute respiratory syndrome (SARS) in 29 countries in 2003;

- high rates of hepatitis $\mathrm{C}$ and hepatitis $\mathrm{B}$;

- a rise in HIV notifications in NSW;

- increasing rates of unprotected anal intercourse and sexually transmissible infections in men who have sex with men;

- increasing rates of chlamydia in young adults;

- a halt in the decline of rates of tuberculosis;

- cyclical outbreaks of pertussis every three or four years;

- repeated importation of measles cases by overseas travellers and the risk of further transmission of measles to contacts in Australia;

- public concern about the incidence of meningococcal disease;

- high rates of foodborne illness and increasing reports of salmonellosis;

- outbreaks of cryptosporidiosis every four or five years, often linked to contaminated swimming pools;

- outbreaks of mosquito-borne disease (mainly due to Barmah Forest virus and Ross River virus infections);

- increasing incidence of organisms resistant to antibiotics;

- the threat of bio-terrorism in NSW, including the spate of exposures, in late 2001, to white powders that potentially contained anthrax.

\section{PRIORITIES}

The following is a list of priorities (in no particular order) for the public health network for communicable disease control in NSW from 2003. Included for each are the major strategies for achieving the priority area, and the draft indicators that can be monitored to assess how well each strategy is implemented. These indicators, when finalised, will be reported annually in the NSW Public Health Bulletin's annual communicable diseases Year in Review. Realising these priorities is dependent on NSW Health maintaining a strong network of skilled public health professionals. The proposed 'top 10' priorities for communicable disease surveillance, prevention and control in New South Wales, from 2003, are:

Disease outbreaks, including those caused by emerging diseases and the deliberate release of biological agents

Strategies

- revise the NSW Notifiable Diseases Database;

- provide training for public health staff in outbreak investigation and control;

- improve liaison between public health staff and clinical laboratories;

- maintain a functioning public health network for sharing information advice.

\section{Indicators}

- number of statewide training opportunities for public health staff;

- number of disease outbreaks investigated.

Blood-borne infections (focussing on HIV, hepatitis C, and hepatitis B)

Strategies

- maintain high quality surveillance data on the incidence and risk factors for HIV;

- educate communities about risk and prevention;

- maintain and build partnerships with affected communities;

- provide needles and syringe programs;

- support universal hepatitis B vaccination of all children and targeted vaccination of risk groups;

\section{Indicators}

- number of needles and syringes distributed in NSW;

- percentage of neonates born to HBV (hepatitis B) positive mothers who are administered HBIG (hepatitis B immunoglobulin) within 12 hours;

- number of newly diagnosed HIV infections by risk group;

- percentage of men who have sex with men who report unprotected anal intercourse;

- percentage of injecting drug users who report sharing injecting equipment;

- number of hepatitis $\mathrm{C}$ notifications;

- number of acute hepatitis B infections.

Sexually transmissible infections (focussing on HIV, chlamydia, syphilis, and gonorrhoea)

Strategies

- monitor the number and characteristics of bacterial sexually transmissible infections through laboratory surveillance;

- enhance surveillance for new syphilis infections;

- educate communities about safe sex;

- improve tracing of contacts of infectious patients. 


\section{Indicators}

- number of new syphilis infections for which enhanced data is available;

- review of contact tracing policy;

- proportion of STI notifications for which contact tracing is reported to have occurred;

- number of babies born with congenital syphilis;

- number of cases of new syphilis infections;

- number of cases of gonorrhoea infections.

Vaccine preventable diseases (focussing on measles, congenital rubella, invasive pneumococcal disease, invasive Haemophilus influenzae type b disease, pertussis, and influenza)

Strategies

- maintain high quality surveillance to monitor the incidence and risk factors for measles, congenital rubella, invasive pneumococcal disease, invasive Haemophilus influenzae type b (Hib) disease, pertussis, and influenza;

- maximise the number of children fully immunised according to the standard childhood immunisation schedule;

- identify and protect susceptible people exposed to measles, Hib disease and pertussis;

- encourage travellers to immunise routinely against measles, mumps, and rubella;

- encourage adults to be vaccinated by facilitating free vaccination against influenza for all persons aged over 65 years;

- screen and, where necessary, vaccinate health care workers against occupationally transmissible infections.

\section{Indicators}

- proportion of children fully immunised at 12 months to less than 15 months;

- 80 per cent of people aged over 65 years immunised against influenza;

- the percentage of Aboriginal and Torres Strait Islanders people immunised against influenza and pneumococcal disease;

- number of measles cases;

- number of cases of vaccine preventable diseases in Aboriginal and Torres Strait Islander persons aged over 50 years;

- number of area health services that report implementation of occupational screening and vaccination policy.

\section{Tuberculosis}

Strategies

- collect and analyse data on the incidence, risk factors, and molecular epidemiology of tuberculosis cases, and the outcome of case treatment;
- provide high quality counselling and treatment services;

- identify, counsel, screen, and treat high risk contacts of infectious cases;

- detect early cases in health care workers.

\section{Indicators}

- release of revised tuberculosis control policies;

- number of tuberculosis cases notified by region of birth, age group, indigenous status, and HIV status.

\section{Enteric disease}

Strategies

- develop a better system for gathering summary data on the incidence, extent, and risk factors contributing to outbreaks of enteric disease;

- work with the food safety authority to improve community education about safe food handling and hand-washing.

\section{Indicators}

- release of revised policies for enteric disease investigation;

- proportion of reported outbreaks of enteric disease investigated using epidemiological methods.

\section{Meningococcal disease}

Strategies

- collect and analyse enhanced surveillance data on the risk factors and management of meningococcal cases in NSW;

- communicate accurate information to close contacts of cases, the public, and health care workers, about the signs, symptoms, and management of the disease;

- identify and protect close contacts of cases of meningococcal disease.

\section{Indicators}

- proportion of school-aged children vaccinated against meningococcal $\mathrm{C}$ disease;

- incidence of meningococcal type $\mathrm{C}$ disease in people aged less than $<20$ years.

\section{Mosquito-borne diseases caused by flaviviruses}

\section{Strategies}

- identify through laboratory surveillance and rapidly investigate possible cases of Murray Valley encephalitis and Kunjin virus infection;

- monitor the risk for Murray Valley encephalitis through regular serological monitoring of sentinel chicken flocks in western NSW;

- communicate prevention advice to people living in or travelling to epidemic areas.

\section{Indicators}

- number of chicken flocks used to monitor Kunjin and Murray Valley encephalitis; 
- number and location of notifications of Kunjin and Murray Valley Encephalitis infections.

Infections caused by antibiotic resistant organisms Strategies

- monitor multi-drug resistant tuberculosis (MDR-TB), methicillin resistant Staphylococcus aureus (MRSA) and vancomycin resistant enterococci (VRE) and Vancomycin Intermediate Staphylococcus aureus (VISA) infections in public hospitals;

- review the public health issues related to the emergence of antibiotic resistance.

Indicators

- report on the public health issues related to antibiotic resistance;

- reports of MRSA, VRE, and VISA in public hospitals.

\section{Infections associated with health care}

Strategies

- review statewide infection control, and the infectioncontrol-related aspects of sterilising services;

- review data collected under the Infection Control Program Quality Monitoring Indicator initiative;
- develop a mandatory routine system for monitoring local compliance with the Infection Control Policy.

\section{Indicators}

- release a NSW Statement of Strategic Directions for Infection Control and revision of the Infection Control Policy;

- incidence of infections and exposures targeted under the Infection Control Program Quality Monitoring Indicator system.

Comments on this list of communicable diseases control priorities are welcome, and should be made to the Director, Communicable Diseases Branch, Locked Mail Bag 961, North Sydney, NSW, 2059.

\section{ACKNOWLEDGMENT}

Thanks to Barbara Telfer, Andrew Marich, Cath Murphy, and Kris Hort for their contributions to this article. 